\title{
Phase Masks for Electron Vortex Beams
}

\author{
Rodney Herring, Adam Schuetze and Elaine Humphrey
}

Advanced Microscopy Facility, Center for Advanced Materials and Related Technology (CAMTEC), Mechanical Engineering, University of Victoria, Canada V8W 3P6

The use of electron vortex beams for SEM and (S)TEM imaging and analytical electron microscopy will have a revolutionary impact on scientific discoveries and technical developments by enabling researchers to image in new ways, make new types of measurements and manipulate the electrons and atoms in materials using its associated orbital angular momentum (OAM) in a manner never before possible [1]. Essential for creating the electron vortex beams is a phase mask that the electron beam must pass through in order to obtain the spiral phase shift of its wavefront. To this end the phase mask needs to contain a structure that will be responsible for creating the wavefront phase shift of the electron beam, preferably by $\mathrm{n} 2 \pi$. A helical phase plate created by a spiraling surface relief structure [2] could be used but it would have several problems such as its difficulty to fabricate with nanometer precision, internal Bragg and diffuse scattering of the electron beam and surface contamination that would distort its shape. Alternatively, electron vortex beams have been created in the TEM by passing the electron beam through a dislocated hologram [3, 4]. A focused ion beam (FIB) can produces a nicely shaped grid pattern in a controlled manner, however, the periodic spacing, $\mathrm{d}$, of the grid is difficult to make fine resulting in low angle diffracted beams $(\theta=\lambda / \mathrm{d})$. Creating phase shifted beams from a crystal with a dislocation is also possible [5] with the advantage of producing high angles of diffraction useful for separating the diffracted beams, however, using a dislocated crystal also has many experimental difficulties including the production of diffuse scattering of the electrons, radiation damage effects that may change the nature of the dislocation, the possible build-up of surface contamination, as well as, the difficulty of finding a pure edge dislocation without a screw component where the screw component will give some wavefront distortion due to the surface relief associated with its core. All for these will induce unknown phase shifts to the electron vortex beams. Thus creating a dislocated phase mask from a thin film of material with known properties using the FIB has the most advantages, however, making a good dislocated phase mask with a fine grating and complex dislocation core shape using the FIB has many challenges, which is the subject of this abstract.

Several types of thin films processed by the FIB were investigated. Substrates for metal epilayers included amorphous carbon, formvar and silicon nitride, the first two in an attempt to produce inexpensive phase gratings didn't produce good results. Metal epilayers of Pt, Au and $\mathrm{Au}-\mathrm{Pd}$ from $10 \mathrm{~nm}$ to $500 \mathrm{~nm}$ thickness were used. Various recipes of FIB cutting were also investigated. The biggest problem was grating collapse, Fig. 1a, which occurred for thick and thin epilayers using multiple passes by the FIB. Au-Pd epilayers on both sides (low 10s nm) of $\mathrm{SiN}(100 \mathrm{~nm})$ produced promising results but the half grating, which is a cantilever beam, was unstable, Fig. 1b and Fig. 1c. Few FIB passes that considered the gouging nature of the Ga ion beam produced the best results, Fig. 1d and Fig. 2. Detailed successful recipes will be presented. 1. R.A.Herring, A New Twist for Electron Beams Science 331 (2011) 155.

2. M. Uchida, A. Tonomura, Generation of electron beams carrying orbital angular momentum Nature 464 (2010) 737. 
3. J. Verbeeck, H. Tian, P. Schattschneider, Production and application of electron vortex beams Nature 467 (2010) 301.

4. B.J. McMorran, A. Agrawal, I.M. Anderson, A.A. Herzing, H.J. Lezec, J.J. McCllelland, J. Unguris, Electron Vortex Beams with High Quanta of Orbital Angular Momentum Science 331, (2011) 192.

5. R.A. Herring, G. Pozzi, Electron Holography using Diffracted Electron Beams (DBH), Introduction to Electron Holography, Eds. E. Volkl, L. F. Allard, D. C. Joy, Kluwer Academic/Plenum Publishers, New York (1999) 295.
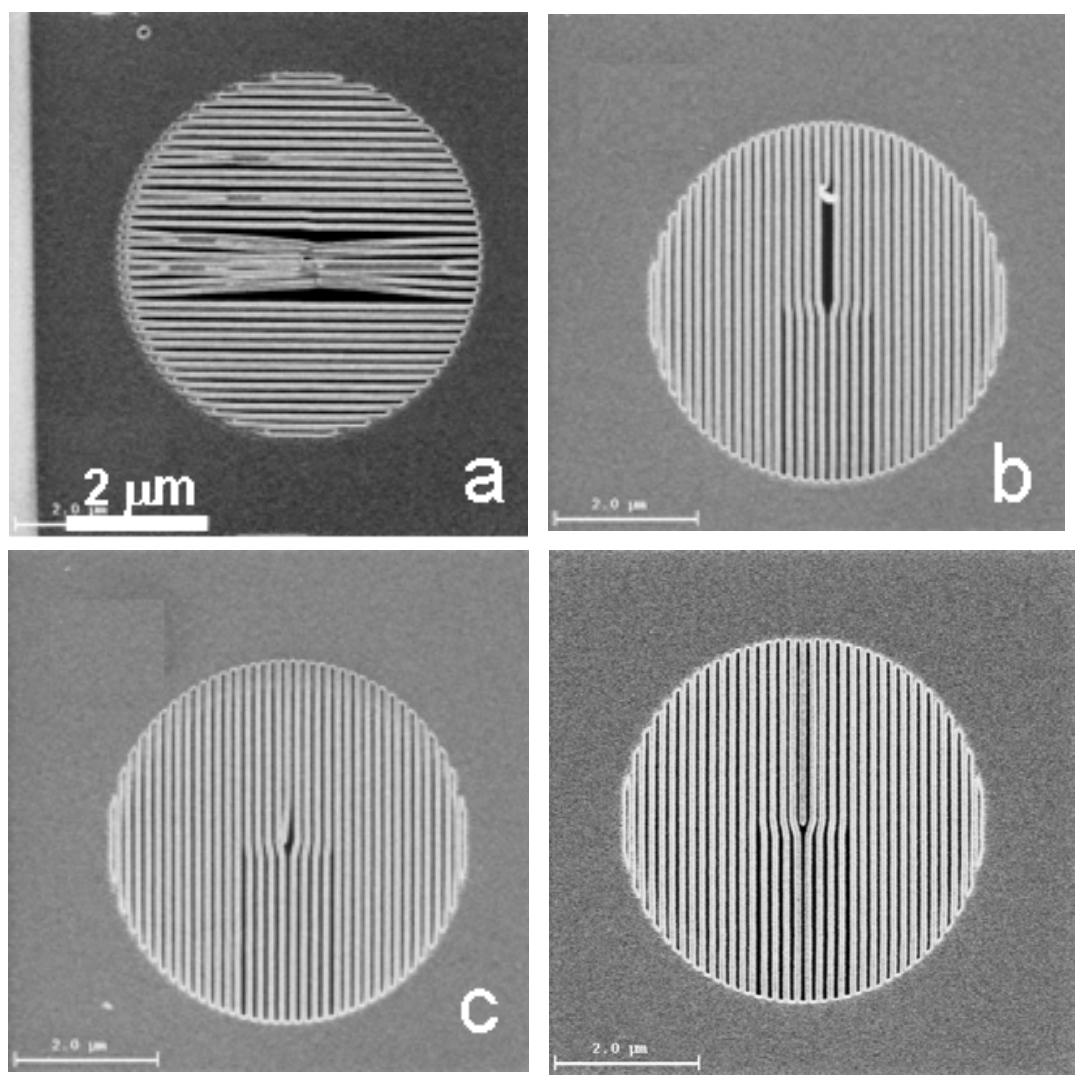

Fig. 1 Dislocated holograms produced by FIB processing of Au-Pd epilayers on Silicon Nitride showing in a) grating collapse, b) and c) half grating curling and d) a perfect phase mask having $139 \mathrm{~nm}$ periodicity sufficient for separating the electron vortex beams for aperturing.

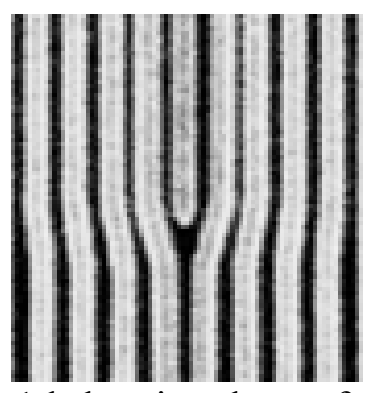

Fig. 2 A higher magnification of Fig. 1d showing the perfect dislocation core region that can be used to produce $\mathrm{a} \pm 2 \pi$ phase shift of the first order electron vortex beams. 